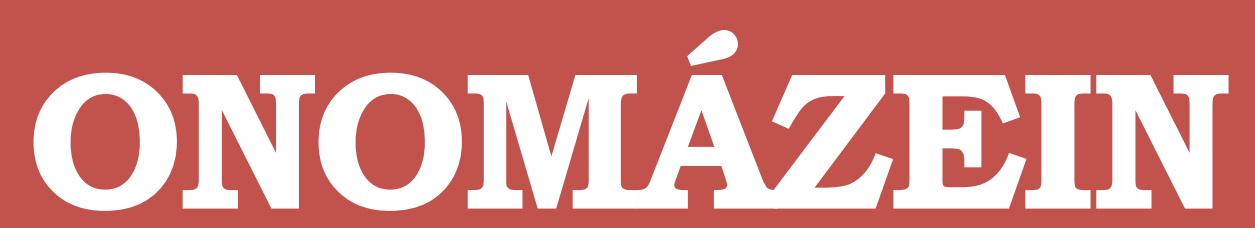

Revista de lingüística, filología y traducción
PONTIFICIA UNIVERSIDAD CATÓLICA DE CHILE FACULTAD DE LETRAS

\title{
Presencia de anglicismos en el campo de las ciencias farmacéuticas
}

Presence of anglicisms in the field of pharmaceutical science

\section{Carmen Luján-García}

Universidad de Las Palmas de Gran Canaria España

\section{(C) $($ (i) $\ominus$}

Carmen Luján-García: Departamento de Filología Moderna, Universidad de Las Palmas de Gran Canaria, España. | E-mail: carmen.lujan@ulpgc.es 


\section{Resumen}

El presente artículo examina el uso de anglicismos dentro del campo de la farmacia, que no parece estar exento del uso de extranjerismos. Con tal fin, se ha elaborado un corpus extraído de tres revistas de este campo, entre otras fuentes, para posteriormente analizar los distintos tipos de anglicismos (no adaptados, adaptados, híbridos y calcos). Los resultados revelan que las unidades léxicas anglicadas sin adaptación o puras son las empleadas con mayor frecuencia, seguidas de los calcos, híbridos y, por último, de los anglicismos adaptados. En un estadio posterior, se ha realizado un doble análisis del corpus: en primer lugar, atendiendo al grado de tecnicidad de los términos, distinguiendo entre anglicismos técnicos, semitécnicos y de uso general, y, en segundo lugar, teniendo en cuenta el campo semántico al que pertenecen los términos (biosanitario, procesos de fabricación/distribución y controles de calidad, TIC y términos generales). Para determinar las motivaciones que conducen al empleo de estos términos, se hace referencia a las funciones pragmáticas de los anglicismos (referencial, textual y expresiva).

Palabras clave: anglicismos; farmacia; medicina; terminología; técnico.

\section{Abstract}

This piece of research intends to examine the use of anglicisms within the field of pharmaceutical science, which does not seem to be exempt from the use of foreign words. Bearing that aim in mind, a corpus has been excerpted from three different journals of this field, among other sources. Secondly, the corpus has been broken down according to different kinds of anglicisms (non-adapted, adapted, hybrids and calques). The findings reveal that the presence of non-adapted or pure lexical units is higher than that of the calques, hybrids and adapted anglicisms. In a lower hierarchy, two analysis of the corpus have been carried out: firstly, discerning different degrees of speciality or technicality distinguishing among technical, semitechnical and general use terms, and, secondly, considering different semantic fields (pharmacy/medicine area, business and marketing, manufacturing/distributing process and quality controls, IT and general terms). Eventually, in order to determine the reasons that lead to the employment of these anglicisms in Spanish, a threefold distinction has been made examining their pragmatic function (referential, textual and expressive).

Keywords: anglicisms; pharmacy; medicine; terminology; technical. 


\section{Introducción}

El campo de la farmacia y la farmacología, al igual que el de otros muchos terrenos de especialización, se caracteriza por la presencia de un tipo de lenguaje que oscila entre lo técnico, pasa por lo semitécnico y cuenta también con vocabulario de la vida cotidiana. Hace ya algo más de una década, Alcaraz Varó (2006) publicó un trabajo que, como su título indicaba, llevaba a cabo una aproximación al lenguaje de la farmacia. Este autor pretendía, de un lado, examinar el tipo de lenguaje empleado en el campo de la farmacia y, de otro, fijar las bases para la elaboración de un diccionario de la ciencia farmacéutica. En este terreno y más allá del estudio de Alcaraz Varó (2006), apenas han visto la luz más trabajos que aborden este tema en el campo de la farmacia. En el dominio afín de la medicina, aunque no demasiado proclive tampoco al análisis en términos lingüísticos, sí que se cuenta con algunas interesantes contribuciones (Arias, 1999; Navarro, 2001; Segura, 2001; Alcaraz Ariza, 2002; Navarro, 2002; Alcaraz Varó, 2008; Neumann, 2013; Acosta Artiles y Bolaños Medina, 2017) que han arrojado luz sobre el empleo de extranjerismos, en particular de anglicismos, en este campo de la ciencia.

Como se puede esperar de un trabajo de esta índole, existe una interdisciplinariedad que abarca tanto a la lingüística como a la ciencia farmacéutica. De ahí que el foco del análisis sea el lenguaje técnico de los profesionales del mundo de la farmacia. En este caso, podemos hablar de un tipo de lenguaje plagado de términos de origen grecolatino, que confieren un aire de seriedad y prestigio y que, dados los mecanismos de formación de términos del español, son los más frecuentes en las ciencias. El dominio de la farmacia y de la medicina está plagado de unidades léxicas de este tipo. Así, por ejemplo, siglas como AL, que proviene de 〈Ad libitum〉, y que significa "como se desee”; AS <Auris Sinistra〉, que significa "oído izquierdo", o bien BD/BID <Bis in Die> "dos veces al día"; BS 〈Blood Sample> o "muestra de sangre" son empleadas con relativa frecuencia por los profesionales del campo que nos ocupa. En lo que se refiere al empleo de palabras más populares o de origen anglosajón, estas son escogidas en múltiples ocasiones, frente a sus equivalentes más formales, pese a que ambos vocablos aparezcan en cualquier diccionario del inglés. Por ejemplo, el término drug es, con toda probabilidad, más empleado por un hablante del inglés, frente a su equivalente más formal o aristocrático, medicine o medicament. De la misma manera, se puede afirmar que el término pill será escogido sin ningún tipo de dudas frente a otros vocablos como bolus o capsule, que tienen un origen grecolatino.

Por otro lado, no debemos olvidar que el inglés se ha convertido en la lengua franca de la comunicación internacional por excelencia. De ahí que no resulte extraño que en la mayoría de los campos de nuestra vida cotidiana encontremos anglicismos y siglas que provienen del inglés (Alcaraz Varó, 2000: 14). Tal como señala este mismo autor (Alcaraz Varó, 2006: 347), existen tres razones que invitan al uso de anglicismos en este campo: 1) la brevedad de las palabras de origen anglosajón, 2) la precisión y 3) la nivelación lingüística que hace que se trate de términos aceptados en gran parte de las lenguas de cultura. Sin duda, esto favorece 
la univocidad deseable en el lenguaje de especialización, aunque, como bien indica Alcaraz Varó, muchas veces más deseable que alcanzable. Todo ello implica que estamos ante una lengua que no solo tiene un gran peso per se, sino que además es un idioma muy extendido en el entorno académico y profesional internacional.

Por consiguiente, la presencia de anglicismos en el campo de la farmacia, como en otros muchos terrenos, parece ser notoria (Alcaraz Varó, 2006: 347) y en el presente trabajo se pretende indagar cuáles son algunos de los términos empleados entre los profesionales hispanohablantes del terreno farmacéutico.

\section{Objetivos}

Este trabajo tiene por objeto analizar la presencia de la lengua inglesa en el campo de las ciencias de la farmacia. Se parte de la hipótesis de que existe presencia de anglicismos en el campo objeto de análisis, así como en otros afines como la medicina, tal como han demostrado trabajos anteriores (Alcaraz Ariza, 1998; Alcaraz Varó, 2006). Dicho análisis busca aportar datos cualitativos actuales y concretos que desvelen tal presencia. Para ello, se han formulado las siguientes preguntas de investigación:

- ¿Qué tipo de anglicismo es el más frecuente en el campo de las ciencias farmacéuticas: no adaptados, adaptados, híbridos o calcos?

- ¿Qué tipo de términos se pueden distinguir? ¿Terminología técnica, semitécnica o bien vocabulario general usado frecuentemente en este campo de especialidad?

- ¿Qué campo semántico es más prolífico al empleo de anglicismos: ciencias biosanitarias; negocios y marketing; procesos de fabricación/distribución y controles de calidad; campo de las TIC o términos generales?

- ¿Qué función cumple el empleo de estos anglicismos en este campo de especialidad?

\section{Método}

Para la compilación del corpus objeto de este análisis, se consultaron varias fuentes de divulgación dentro del campo de la farmacia. Se escogieron tres revistas. Una de ellas es www.correofarmaceutico.com, en su edición digital. Esta es una publicación que se inició en 2000 y es visitada por más de 35.000 usuarios al mes (OJD), y, según sondeos anuales de varios institutos de opinión, es considerada la principal y más influyente fuente de información de los farmacéuticos españoles, tal como afirma la web de la propia revista, que está editada por Unidad Editorial, primer grupo español de prensa especializada. La segunda revista analizada fue www.elfarmaceutico.es, también en formato digital. Se trata de un producto de Ediciones Mayo que, como indica la web de la revista, "dedica sus esfuerzos desde hace más 
de 30 años a la formación e información de los farmacéuticos”. Se trata de una revista que pretende actuar como publicación científica en el ámbito de la farmacia, con contenidos que abarcan aspectos teóricos y prácticos de la salud, así como de reflexión y debate crítico de la profesión. Su periodicidad es quincenal y se publican 20.000 ejemplares en formato papel por número, lo que puede indicar que se trata de un referente de la profesión. Por último, la última revista consultada fue www.infarmacias.es, que, como indica su portal, se trata de una revista especializada dirigida a las oficinas de farmacia, laboratorios, fabricantes, colegios farmacéuticos, cooperativas, grupos de distribución, asociaciones y a todas las entidades que engloban el sector farmacéutico.

El método se basó en la compilación de anglicismos empleados en el campo objeto de estudio usando distintas fuentes. En primer lugar, se extrajo el corpus de las tres mencionadas revistas del ámbito de la ciencia farmacéutica. El proceso de extracción se llevó a cabo de forma manual, es decir, por un lado, leyendo artículos de las revistas y extrayendo los anglicismos presentes en estos y, por otro lado, se utilizó el buscador de las propias revistas en línea, en el que se insertaba una unidad léxica anglicada y, de forma automática, el sitio web aportaba los artículos que contenían ese término anglicado. Estas revistas aportaron un listado de noventa anglicismos.

Una vez conformado el corpus objeto de estudio, se procedió a contrastarlo con varias fuentes lexicográficas tales como el Diccionario terminológico de ciencias farmacéuticas (inglésespañol, Spanish-English) de Alcaraz, Domínguez y Martínez (2007), el Gran diccionario de anglicismos (Rodríguez González, 2017), el Diccionario de la lengua española (DLE), el Glosario de medicamentos: desarrollo, evaluación y uso de Arias (1999) y el glosario que ofrece el artículo "Palabras y expresiones inglesas de traducción difícil o engañosa en investigación clínica, bioestadística y medicina basada en la evidencia” de Navarro y González de Dios (2014). Estas fuentes aportaron, en muchos casos, la definición del anglicismo objeto de análisis. Otros significados, en cambio, debieron ser consultados en otras fuentes más especializadas (ver tablas 3, 4, 5 y 6 en el anexo).

A continuación, se procedió a documentar los anglicismos del corpus buscando ejemplos donde estas unidades léxicas fueran usadas en el contexto de alguna de las revistas farmacéuticas arriba señaladas. De esta forma, se podía confirmar si, en efecto, se emplean esos vocablos o no dentro del ámbito de las ciencias farmacéuticas. Teniendo en cuenta que con este trabajo se pretende un análisis de anglicismos, se comenzará por incluir la definición de anglicismo del DLE: "giro o modo de hablar propio de la lengua inglesa".

Para la posterior selección de distintos tipos de anglicismos se empleó la categorización creada por Furiassi, Pulcini y Rodríguez González (2012: 6) al tratarse de una de las más recientes y, probablemente, de la más extendida en la actualidad. Esta categorización (ver figura 1) distingue grosso modo préstamos directos e indirectos. Dentro de los directos se 
pueden diferenciar híbridos, falsos préstamos —también llamados pseudopréstamos-y préstamos per se, que pueden ser, a su vez, adaptados y no adaptados. Este tipo de préstamos directos son de carácter léxico. A la derecha de la figura 1 se observan los préstamos indirectos, dentro de los cuales podemos distinguir los calcos y los préstamos semánticos. Dentro de los calcos, distinguiremos entre préstamos traducidos, préstamos versionados y préstamos creados. Los préstamos indirectos son de carácter sintáctico y afectan más a la estructura de la oración que los léxicos en la lengua receptora.

\section{FIGURA 1}

Categorización de anglicismos de Furiassi, Pulcini y Rodríguez González (2012: 6)

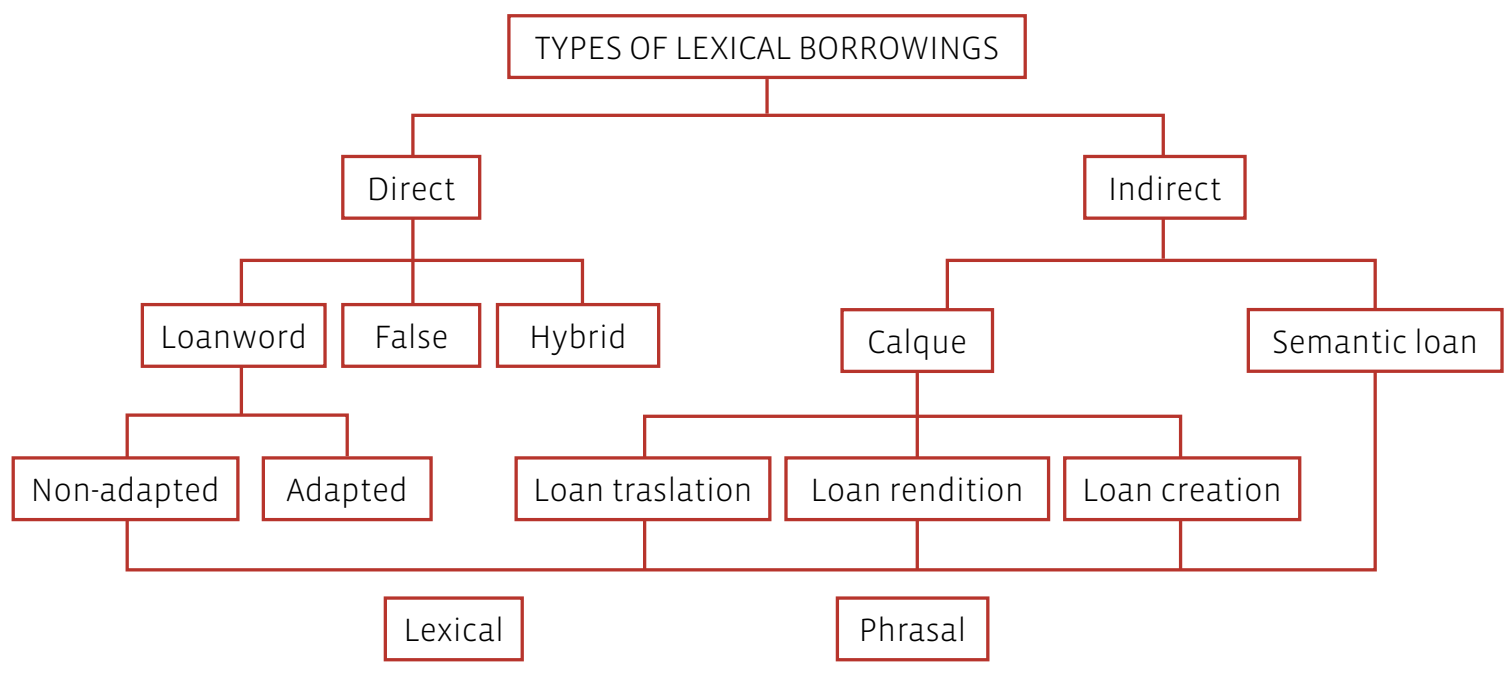

En el presente estudio, debido a la naturaleza del corpus compilado, se procedió a adaptar esta categorización, que se centra únicamente en las cuatro categorías que se enumeran a continuación. Como se puede observar en el siguiente epígrafe de resultados, los tipos de anglicismos que se analizaron fueron los siguientes:

$\rightarrow$ Préstamos anglicados o anglicismos no adaptados.

$\rightarrow$ Préstamos anglicados o anglicismos adaptados.

$\rightarrow$ Préstamos anglicados o anglicismos híbridos.

$\rightarrow$ Calcos (traducciones de préstamos).

En este trabajo, además de llevar a cabo un análisis de los distintos tipos de anglicismos hallados en las fuentes examinadas, que es uno de los propósitos principales de este estudio, se consideró pertinente analizar otros aspectos tales como un desglose por nivel de especialización, debido a que se trata de un lenguaje profesional con un elevado nivel de especialización, y otro desglose por campos semánticos, al tratarse de terminología que se encuentra tanto 
dentro del terreno de la farmacia como en otros dominios afines. Por tanto, este estudio se centra en el análisis de anglicismos, pero en un mayor nivel de análisis se examina el grado de especialización de estas unidades léxicas anglicadas, así como de los campos semánticos a los que pertenecen, como se puede observar en la sección de resultados.

Debemos señalar que durante el proceso de compilación del corpus se hallaron numerosas siglas anglicadas que se emplean en este campo. Sin embargo, dado el elevado número de estas y las limitaciones de espacio del presente estudio, se decidió abordar con profundidad la presencia de siglas en otro trabajo (Luján-García, 2020). En el siguiente epígrafe, se procederá al análisis de los resultados obtenidos.

\section{Resultados}

\subsection{Anglicismos}

Tomando en consideración la adaptación de la categorización propuesta en el epígrafe anterior, en esta sección se procederá a examinar los resultados obtenidos. En el presente trabajo hemos hallado un total de 90 términos que tienen una relación más directa o indirecta con el terreno de la farmacia. En este análisis, en primer lugar, haremos un desglose global de los anglicismos que forman parte de nuestro corpus (ver tabla 1 ).

TABLA 1
Desglose de variedad y porcentaje de anglicismos
\begin{tabular}{ccc} 
TIPOS DE ANGLICISMOS & VARIEDAD DE ANGLICISMOS & $\%$ \\
\hline No adaptados & 69 & 76,7 \\
\hline Adaptados & 2 & 2,2 \\
\hline Híbridos & 6 & 6,6 \\
\hline Calcos & 13 & 14,5 \\
\hline Total & 90 &
\end{tabular}

Como se desprende de la tabla 1, el mayor porcentaje de unidades léxicas anglicadas se da entre los anglicismos que no han experimentado ningún tipo de adaptación al español, con un $76,7 \%$ del total. En segunda posición, con un $14,5 \%$ del total, se hallan los calcos del inglés al español. En tercer puesto se encuentran los híbridos o combinados compuestos por una unidad léxica en español y otra en inglés con un 6,6\% del total. Por último, encontramos los dos únicos casos de anglicismos adaptados (emblistado y randomizado), que equivalen a un 2,2\% del total. A continuación, se procederá a un análisis más pormenorizado del corpus de este estudio. 


\subsubsection{Anglicismos no adaptados (sesenta y nueve términos y expresiones)}

Este tipo de anglicismo no presenta ninguna alteración de tipo ortográfica en su traslado del inglés a la lengua receptora, el español, y, tal como refleja la tabla 1 , ofrece el mayor número de términos. A este tipo de anglicismos también se les conoce como "puros" o "crudos", si atendemos a la terminología de Lorenzo (1987: 77-79).

En las siguientes líneas incluimos el listado de términos de las revistas examinadas donde se emplean algunas de estas unidades léxicas.

App, area manager, benchmarking, big data, blister pack, blog, blue-box, business development manager, business unit, business unit manager, checklist, checkpoint, clinical research assistant, clinical trial management, cross docking, customer journey, data matrix, data mining, endpoint, export manager, gold standard, good clinical practices, government affairs manager, hashtag, hazard ratio, items, joint venture, key account manager, kit, lock-up, low dose, market access manager, non-sense, odds ratio, one-to-one, open source, ophthalmological tested, partner, peer monitoring, pellets, pipeline, pivotal, premium price, pricing, prime time, product manager, public affairs manager, quality and compliance manager, ranking, ready-made, real-world evidence, regulatory, reimbursement, retail, screening, senior consultant, senior product manager, software, source data, stakeholder, stand, start-up unit, stock, supply chain, test, trade marketing, unit dose, waterproof y water resistant.

Como se puede observar en el listado anterior, existe una gran variedad de términos asociados al campo de la farmacia que van desde el sector empresarial al de la medicina pasando por vocabulario del entorno TIC, pues debemos tener presente que el farmacéutico es un empresario y además de eso gestor y experto del medicamento, por lo cual existe una vinculación de esta figura profesional con tan variados sectores.

A continuación, aportaremos algunos ejemplos concretos de estos anglicismos usados en contexto en las distintas revistas consultadas. No obstante, en la tabla 3 del apéndice se puede consultar el listado completo de anglicismos no adaptados examinados usados en contexto. Conviene aclarar que, aunque los términos aparecen en negrita en este trabajo con el objeto de resaltarlos para su rápida identificación, en el original no necesariamente aparecían con algún tipo de marcador, como se abordará más adelante en este artículo (sección 4.1.5).

El benchmarking, también definido como una medida estándar a la que una actividad, rendimiento, servicio o resultado se puede comparar, constituye una práctica actual en farmacia. Prueba de ello es el siguiente titular:

(1) ‘Benchmarking’: cómo mejorar observando a otros (correofarmaceutico, 04/07/2018). 
(2) Según la experta de IMS, existen tres tipos de benchmarking, el interno, el funcional y el competitivo (correofarmaceutico, 04/07/2016).

Según el Gran diccionario de anglicismos (GDA), un blister pack es un "envase para manufacturados pequeños en soporte de cartón o cartulina”. Como cabría esperar, se trata de un término también empleado en farmacia, como demuestra el siguiente ejemplo 3.

(3) Zaragozá también habló de investigar para desarrollar nuevas formas de administración que mejoren la toma de los medicamentos. "Tenemos blísters imposibles de abrir para una persona mayor y antipsicóticos muy difíciles de tomar por los enfermos”, constata (correofarmaceutico, 01/03/2018).

Otro uso llamativo es el del término cross-docking o sistema de distribución donde las unidades logísticas son recibidas en una plataforma de alistamiento y no son almacenadas, sino preparadas para ser enviadas de la manera más inmediata. No es un término exclusivo del terreno farmacéutico, sino más del mundo empresarial, aunque empleado regularmente en farmacia. El siguiente ejemplo 4 lo ilustra.

(4) Es una plataforma de cross-docking: Ios pedidos llegan de los laboratorios y se distribuyen a todos los almacenes del Grupo en cuestión de horas (imfarmacias, 24/11/2016).

Data matrix es un anglicismo empleado para referirse a lo que el siguiente ejemplo 5 describe.

(5) Además, a efectos de codificación, el CN quedará incorporado a la información contenida en el código de producto incluido en el código de barras bidimensional (también denominado código datamatrix) (correofarmaceutico, 13/07/2017).

Joint venture o formación de una sociedad o alianza entre varias compañías o laboratorios para la producción de un determinado producto es otro anglicismo no exclusivo del campo de la farmacia, pero empleado en este terreno, tal como demuestra el ejemplo 6.

(6) Aunque la compañía se estableció en España en 1993 a través de una joint venture con Laboratorios Cusí, S.A., no fue hasta 1995 que comenzó su actividad bajo el nombre de LEO Pharma (elfarmaceutico, 14/12/2015).

Low dose es, en cambio, un anglicismo técnico sin adaptación dentro del terreno de la farmacia y se emplea para referirse a una dosis baja de algún componente en un medicamento, como se ilustra en el ejemplo 7.

(7) Isdin lanza Itragerm 50mg, el primer itraconazol low dose con biodisponibilidad mejorada (infarmacias, 12/11/2014). 
Otro ejemplo de término técnico o especializado en el campo de la farmacia es pellet y hace referencia a una forma farmacéutica sólida preparada para su implantación debajo de la piel con el fin de obtener una absorción lenta y sostenida.

(8) Los productos estrella de la fábrica maña, que es referencia dentro del grupo en tecnologías de pellets (cápsulas con gránulos), son el omeprazol, el lansoprazol, la atorvastatina y el pantoprazol (correofarmaceutico, 11/10/2014).

Unit dose hace referencia a un método de empaquetado y administración de medicación a través de dosis individuales y es un término especializado dentro del campo de la farmacia. El ejemplo 9 es prueba de ello.

(9) El término unit dose drug packaging se debe traducir por medicamentos envasados en dosis unitarias, y nunca por el término equivocado de unidosis (correofarmaceutico, 28/06/2010).

Para consultar más ejemplos concretos de estos anglicismos usados en contexto, se puede consultar la tabla 3 en el anexo.

\subsubsection{Anglicismos adaptados (dos términos)}

Dentro de este tipo de anglicismos, se han encontrado dos términos: emblistado, que proviene del inglés blister pack o tipo de envasado pequeño en el que se envasan medicamentos. Se trata de una adaptación ortográfica y fonética donde el sustantivo inglés blíster recibe un prefijo -em que lo convierte en verbo emblistar y, por último, un sufijo -ado que convierte a este verbo en su forma de participio (ver ejemplo 10).

(10) La industria está haciendo grandes esfuerzos por mejorar sus procesos. Un ejemplo es la introducción de la última tecnología en el emblistado de comprimidos de Cinfa (correofarmaceutico, 02/03/2017).

El segundo caso es el de randomizado, que proviene del anglicismo random, al que se le ha añadido el sufijo-ado, con el objeto de verbalizar y adaptar al español este sustantivo (ver ejemplo 11).

(11) También se expondrán los resultados de un estudio en fase III randomizado sobre la eficacia y seguridad comparando atezolizumab, un anticuerpo monoclonal, bien combinado con cobimetinib o en monoterapia con regorafenib, un inhibidor de la tirosina cinasa, en cáncer colorrectal metastásico refractario a quimioterapia (correofarmaceutico, 20/06/2018).

\subsubsection{Híbridos (seis términos)}

Esta categoría abarca el tipo de anglicismo que combina el uso de un vocablo español con otro inglés, creando una formación léxica compuesta. A continuación, nos referiremos a los casos encontrados en este trabajo: 
Farmacia on line u oficina de farmacia que vende medicamentos a distancia, como se puede ver en el ejemplo 12.

(12) Canadá: crece la preocupación por las farmacias ‘on line’ (correofarmaceutico, 05/07/2018).

Gestión de back-up o la realización de una copia de un archivo o fichero por seguridad (ver ejemplo 13).

(13) Protección de Datos exige, en sus artículos 94 y 102, la realización de copias de respaldo y de recuperación de los ficheros automatizados que contengan datos de carácter personal. Una copia de respaldo o backup es la copia de un fichero automatizado en un soporte que posibilite su recuperación (correofarmaceutico, 15/02/2016).

Medicamento Me-Too se emplea para referirse al medicamento aprobado después de un producto pionero y se define como comparable o similar, pero no es un producto clínicamente superior, como puede observarse en el ejemplo 14.

(14) Otra cuestión que debe afrontar la industria es cómo gestionar una cartera en la que hay innovación no coste-efectiva y me-too (correofarmaceutico, 05/02/2007).

Prescripción off label consiste en una práctica de uso de medicamentos fuera de indicación, tanto en lo que ataña a la duración del uso de ese medicamento como en la dosis. A continuación, el ejemplo 15 muestra el empleo de este compuesto híbrido en un titular de una noticia de la revista correofarmaceutico.

(15) El bajo soporte científico pesa sobre la prescripción 'off-label’ (correofarmaceutico, 11/09/2017).

Test de legibilidad o prueba que se realiza para garantizar que un usuario de un medicamento es capaz de encontrar y comprender con facilidad la información de un prospecto. A continuación, podemos ver el ejemplo 16, donde se emplea este híbrido anglicado.

(16) Autorización: la industria, preocupada por los test de legibilidad y el Braille (correofarmaceutico, 09/06/2008).

Consultor senior hace referencia a una persona que hace las funciones de consultoría con una experiencia de varios años en ese terreno. Así, en lugar de consultor experto o con experiencia, se emplea el adjetivo anglosajón senior, como refleja el ejemplo 17.

(17) En resumen, el dispositivo 640G representa el fin de la mitad del camino que lleva hasta el páncreas artificial», destaca el Dr. Ignasi Conget, médico consultor senior de la Unidad de Diabetes del Servicio Endocrinología, en el Hospital Clínic de Barcelona (Elfarmaceutico, 17/04/2015). 


\subsubsection{Calcos (trece tẻrminos)}

En este epígrafe nos centraremos en el análisis de un listado de términos que han sido calcados del inglés.

Comercio paralelo <parallel trade〉; co-pago 〈co-payment 〉; coste-eficiencia <cost-efficiency>; dosis diaria definida 〈defined daily dose (DDD); especificaciones 〈specifications〉; genérico 〈generic〉; implementar <implement>; medicamento huérfano <orphan medicine〉; monitorización <monitoring〉; prescripción fuera de indicación <off-label prescription〉; reportar <report>; Servicio Nacional de Salud <National Health Service〉 y estandarización 〈standardization〉.

Uno de los vocablos es comercio paralelo, que proviene del inglés parallel trade y que puede observarse en el siguiente ejemplo (18) de un titular de revista farmacéutica.

(18) La EMA agrupa en una 'web’ datos del comercio paralelo (correofarmaceutico, 20/07/2015).

El compuesto coste-efectividad constituye una traducción literal de cost-effectiveness o cost-efficiency. El siguiente ejemplo (19) refleja este uso en un artículo de la revista correofarmaceutico.

(19) El objetivo es tener datos sobre el coste-efectividad que puedan ser utilizados en las decisiones sobre precio, reembolso y utilización de nuevos fármacos (correofarmaceutico, 05/02/2007).

Medicamento huérfano es otro ejemplo de calco del inglés orphan medicine. El ejemplo 20 ofrece el uso de este compuesto en la revista correofarmaceutico.

(20) La EMA recomienda acalabrutinib como medicamento huérfano en tres tipos de cáncer (correofarmaceutico, 03/03/2016).

Prescripción fuera de indicación, que procede de <off-label prescription〉, hace referencia a la administración de un medicamento sin tener en cuenta las indicaciones establecidas con el objeto de adaptarse a las necesidades específicas de un determinado paciente. El siguiente titular (ejemplo 21) ilustra este uso.

(21) La Comisión regional de Farmacia murciana garantiza el buen uso de fármacos fuera de indicación a 600 pacientes (imfarmacias, 18/07/2016).

Reportar con el sentido de "informar o dar cuenta a alguien de algo" (DLE) es una nueva acepción con la que se emplea este término en castellano por influencia del inglés. El ejemplo 22 es buena prueba de ello. 
(22) Susana Vilas asume el liderazgo de esta área y se responsabiliza de desarrollar la estrategia integral de comunicación del Grupo, así como de gestionar su imagen corporativa. Vilas forma parte del Comité de Dirección del Grupo Indukern y reportará a Raúl DíazVarela, vicepresidente ejecutivo del Grupo (elfarmaceutico, 08/02/2013).

\subsubsection{Inconsistencias ortográficas}

Como se comentaba anteriormente, todos estos vocablos son usados en las distintas fuentes o revistas farmacéuticas consultadas con numerosas inconsistencias ortográficas, pese a que en este trabajo aparezcan en forma cursiva o negrita en el caso de los ejemplos que nos preceden con el objeto de mostrar con mayor claridad los términos objeto de análisis. En las fuentes originales, podemos, en ocasiones, ver estas palabras entrecomilladas, normalmente con comillas simples, o bien en cursiva, en negrita o bien en redondas, sin ningún tipo de marcador que indique que se trata de un extranjerismo. Hemos incluso observado que en una misma revista el anglicismo puede aparecer con dos formatos distintos (cursiva y sin marcador alguno, por ejemplo) e incluso con tilde o sin ella. La ortografía del vocablo varía, en gran medida, según el criterio de la persona que escriba el artículo. También nos podemos encontrar con que un término compuesto puede aparecer escrito separado o unido: Datamatrix / data matrix; checklist / chek list.

En este sentido, procede incluir algunas ilustraciones extraídas de las revistas analizadas que permitan observar tales inconsistencias. Así, por ejemplo, hallamos datamatrix donde el término aparece como una única unidad, pero también separado (ver ejemplos 23 y 24), como se comentaba en el anterior párrafo.

(23) Además, a efectos de codificación, el CN quedará incorporado a la información contenida en el código de producto incluido en el código de barras bidimensional (también denominado código datamatrix). (wWw.correofarmaceutico.com, 13/07/2017).

(24) Esteve reconoció que los laboratorios esperan conocer cómo se va a articular "Ia convivencia del Data Matrix con el cupón precinto", teniendo en cuenta que los productos lanzados antes del 9 de febrero de 2019, fecha de entrada en vigor de la nueva normativa, podrán estar en el mercado hasta el momento en que caduquen. Este hecho es uno de los que más preocupan también a las empresas de genéricos, como expresó la directora técnica de Aeseg, Amalia Avilés (www.correofarmaceutico.com, 30/05/2016).

Por otro lado, se incluirán dos ejemplos que también demuestran la falta de consistencia en la ortografía de check list, que aparece unido y separado (ver ejemplos 25 y 26).

(25) En Farmacia Colldeforn han fijado tres indicadores: "El primero tienen en cuenta el margen bruto por operación o rendimiento por ticket de cada empleado. Los checklist miden el porcentaje de calidad de trabajo, y la parte de los servicios valora la cantidad de 
clientes que logramos y la ratio de altas y bajas", revela Corona. Aun así, recuerda que hay empleados que no obtienen los mejores resultados según estos indicadores, pero que destacan por otras cualidades (www.correofarmaceutico.com, 12/06/2018).

(26) Ante una inspección, realizar una 'check list' y disponer de formularios (www.correofarmaceutico.com, 18/11/2013).

En ambos casos se trata de la misma publicación, www.correofarmaceutico.com; sin embargo, no parece existir un criterio uniforme para escribir algunos de estos anglicismos. Podemos, incluso, ser testigos de que en el caso del ejemplo 25 se emplea la cursiva y en el 26 no ocurre lo mismo, sino que se emplean las comillas simples. Tal como señalan De la Cruz y Tejedor (2012: 104), "when the term has not been completely integrated into the system, the foreign word tends to be marked by means of double quotation marks, bold or italics".

Merece también ser mencionado el caso de ophtalmological tested, que aparece con un error ortográfico en la publicación (ver ejemplo 27), ya que omite la segunda -h que daría lugar a la forma correcta Ophthalmological tested.

(27) 10. No pica en los ojos (ophtalmological tested) (www.elfarmaceutico.es, 16/03/2015).

Esto, como cabe esperar, implica que al tratarse de extranjerismos que se usan en el español, o bien de forma transitoria objeto de una moda, o bien en proceso de aclimatación a esta lengua, muchos de los usuarios no cuentan con criterios que determinen con precisión cómo resaltar estas palabras.

\subsubsection{Dobletes}

En algunos casos, estos términos son empleados tanto en su versión en inglés como en la versión española. Tal es el caso de vocablos como prescripción off label, que convive con prescripción fuera de indicación. Lo mismo ocurre con la sigla GMP, que en inglés viene a decir Good Manufacture Practice, no presente en este estudio. Sin embargo, la versión en español también se emplea con bastante frecuencia, NCF o Normas de correcta fabricación. Por tanto, hay casos en los que se observa una coexistencia de las dos variantes, la española y la inglesa.

\subsubsection{Desglose de los anglicismos por grado de especialización}

La actual sociedad del conocimiento en la que vivimos se caracteriza por la interdisciplinariedad y la especialización, lo que tiene una estrecha relación con el surgimiento de lo que Alcaraz Varó (2000) denominó "lenguas académicas y profesionales". Se trata de ese tipo de lenguas que emplean grupos de profesionales tales como farmacéuticos, médicos, abogados, entre otros, que usan una terminología específica de su campo de trabajo. 
El léxico científico suele caracterizarse por la precisión, la neutralidad, la economía y el empleo de recursos, con frecuencia gráficos, tales como la apoyatura gráfica de letras del alfabeto latino o griego, o bien siglas, abreviaturas, símbolos químicos (Gutiérrez Rodilla, 2005: 22-26). Teniendo en consideración el grado de especialización del campo que nos ocupa, el de las ciencias farmacéuticas, en el presente epígrafe se ha llevado a cabo un desglose de las unidades léxicas examinadas distinguiendo tres tipos de términos según su grado de especialización. Tal como señala Gutiérrez Rodilla (2005: 29), el vocabulario científico parece disfrutar de una gran movilidad, tanto de forma "horizontal”, pues los términos pasan de un área de conocimiento a otras con el mismo significado o cambiándolo, como de forma "vertical", es decir, pudiendo ser términos superespecializados para llegar a convertirse en palabras utilizadas a diario por cualquier hablante de a pie. De ahí que sea necesario aclarar que los límites entre lo que es puramente técnico o semitécnico, así como entre lo que pertenece exclusivamente al terreno de la farmacia y lo que se solapa con otras ciencias o áreas de especialidad, son muy difusos. En otras palabras, es posible encontrarse con términos técnicos en un contexto semitécnico o bien con un vocablo semitécnico incluso empleado con un significado algo más general. Se pueden dar solapamientos en el uso de este tipo de terminología. No obstante, en este trabajo hemos ubicado las unidades léxicas analizadas dentro de la categoría o contexto en el que son empleados con mayor frecuencia:

\section{TABLA 2}

Desglose de unidades léxicas según grado de especialización

\begin{tabular}{lcc} 
& $N^{\circ}$ DE CASOS & $\%$ \\
\hline Términos técnicos & 18 & 20 \\
\hline Términos semitécnicos & 43 & 47,7 \\
\hline Términos generales empleados en farmacia & 29 & 32,3 \\
\hline Total & 90 & \\
\hline
\end{tabular}

4.1.7.1. Términos y expresiones técnicas (18 casos): Se trata de unidades léxicas empleadas exclusivamente por una determinada comunidad en un terreno específico, fundamentalmente una de las áreas de las ciencias consideradas "duras" (física, química, las matemáticas, la ingeniería, entre otras). Estos términos están sujetos a un alto grado de normalización y se caracterizan por su precisión y univocidad (Martínez-Motos, 2011: 9). En el contexto que nos ocupa, el de la farmacia, hemos hallado los siguientes ejemplos de términos y expresiones que podrían ser consideradas técnicas: blue box, clinical research assistant, clinical trial management, customer journey, endpoint, good clinical practices, low dose, ophthalmological tested, pellet, unit dose, medicamento me-too, prescripción off label, co-pago, dosis diaria definida, genérico, medicamento huérfano, prescripción fuera de indicación y servicio nacional de salud. 
4.1.7.2. Términos y expresiones semitécnicas ( 43 casos): Estas palabras normalmente provienen del lenguaje general pero han adquirido uno o más significados cuando son empleadas dentro de un campo específico (Martínez-Motos, 2011: 9). Por tanto, tienen un componente técnico pero son usadas en otros terrenos o campos de especialización. En nuestro corpus observamos algunas unidades léxicas que no están limitadas al campo de la farmacia, sino que también son empleadas en otras áreas como la del comercio y los negocios o las TIC. Áreas que, tal como indicamos anteriormente, están ligadas al profesional farmacéutico: area manager, benchmarking, big data, business development manager, business unit, business unit manager, checkpoint, cross docking, data matrix, data mining, export manager, gold standard, government affairs manager, hashtag, hazard ratio, joint venture, key account manager, lock-up, market access manager, odds ratio, open source, pipeline, premium price, pricing, product manager, public affairs manager, quality and compliance manager, real-world evidence, reimbursement, retail, screening, senior consultant, senior product manager, source data, stakeholder, start-up unit, supply chain, trade marketing, gestión de back-up, emblistado, randomizado, comercio paralelo y coste-eficacia o eficiencia.

4.1.7.3. Términos y expresiones generales, aunque empleados en farmacia (29 casos): Este grupo se compone de vocabulario general que se usa en un contexto especializado, pero sin perder su significado original. Algunas de estas unidades o expresiones son las siguientes: app, blister pack, blog, checklist, item, kit, one-to-one, non-sense, partner, peer monitoring, pivotal, prime time, ranking, ready made, regulatory, software, stand, stock, test, consultor senior, especificaciones, farmacia on-line, implementar, monitorización, reportar, estandarización, test de legibilidad, water proof y water resistant.

A partir de estos datos, se puede afirmar que las unidades léxicas anglicadas semitécnicas parecen ser las empleadas con mayor frecuencia (47,7\%). Como bien indica Alcaraz Varó (2006: 353), "este vocabulario, que es polisémico, se ha formado en la mayoría de los casos por extensión del significado mediante el proceso de analogía, añadiendo acepciones adicionales al significado tradicional". Se trata en definitiva de palabras que tienen cierto grado de tecnicidad, pero se emplean en variados campos, como puede ser el de la farmacia, la medicina, la economía y/o el comercio.

Los vocablos técnicos también constituyen un 20\% del total. Se trata de términos "cuyos significados quedan definidos de forma unívoca y localización dentro de una teoría" (Alcaraz Varó, 2006: 351). Son palabras que se emplean dentro del campo de la farmacia y cuyos significados son claros, sin prestarse a confusión; de ahí que se consideren monosemias.

También se detectaron unidades léxicas anglicadas de uso frecuente (32,3\%) y que no necesariamente se han de usar solo en el campo de la farmacia. De hecho, se observa en el corpus compilado que estas se usan con mayor o menor frecuencia en el lenguaje general y, como señala Alcaraz Varó (2006: 354), "viven dentro de los aledaños de la especialidad". De esta 
manera, palabras como checklist, item, especificaciones, por mencionar algunos ejemplos, forman parte del habla cotidiana de muchos farmacéuticos, pero también podemos encontrarnos con estos términos en el habla de casi cualquier hablante de a pie del español.

\subsubsection{Desglose de los anglicismos por campos temáticos}

En este epígrafe se hará una distinción de los términos anglicados teniendo en cuenta el campo semántico al que se podrían adscribir las distintas voces analizadas. En este sentido, se distinguirán los siguientes campos:

4.1.8.1. Términos y expresiones pertenecientes a las ciencias biosanitarias: Este campo abarca vocablos relacionados con la medicina, la farmacia y la biología. Las voces que podrían encuadrarse dentro de este campo semántico son: blue-box, clinical research assistant, clinical trial management, endpoint, gold standard, good clinical practices, hazard ratio, low dose, pellet, odds ratio, real world evidence, unit-dose, test, water proof, water resistant, medicamento me-too, ophthalmological tested, pivotal, screening, prescripción off-label, test de legibilidad, dosis diaria definida, genérico, medicamento huérfano, prescripción fuera de indicación, servicio nacional de salud y randomizado. Esto hace un total de 27 términos del corpus (30\%).

\subsubsection{Términos y expresiones pertenecientes al campo de los negocios y el marketing} Dentro de estos campos próximos, hallamos las siguientes unidades léxicas anglicadas: area manager, business development manager, business unit, business unit manager, customer journey, export manager, government affairs manager, joint venture, key account manager, lock-up, market access department/manager, pipeline, premium price, pricing, product manager, public affairs manager, ranking, reimbursement, retail, stakeholder, stand, start-up unit, trade marketing, co-pago, comercio paralelo y coste-eficacia. Se trata de 26 vocablos o, lo que es lo mismo, un $28,8 \%$ del total.

4.1.8.3. Términos y expresiones que no pueden adscribirse a un campo concreto por ser empleados en múltiples procesos de fabricación y distribución y/o controles de calidad: blister pack, benchmarking, checklist, consultor senior, cross docking, item, kit, one-toone, partner, peer monitoring, quality and compliance manager, ready-made, regulatory, senior consultant, senior product manager, stock, supply chain, emblistado, especificaciones, implementar, monitorización, reportar y estandarización. Este campo abarca el mayor porcentaje de los términos analizados, 23 vocablos o un 25,5\% del total.

4.1.8.4. Términos y expresiones pertenecientes al campo de las TIC (tecnologías de la información y la comunicación): app, big data, blog, checkpoint, data mining, hashtag, data matrix, farmacia on line, gestión de back-up, open source, software, source data. Esto supone un total de doce términos, lo que hace un porcentaje de un 13,3\% del total. 
4.1.8.5. Términos y expresiones generales: non-sense y prime time, un porcentaje de 2,2\%.

\section{GRÁFICO 1}

Porcentajes de anglicismos desglosados por campos semánticos

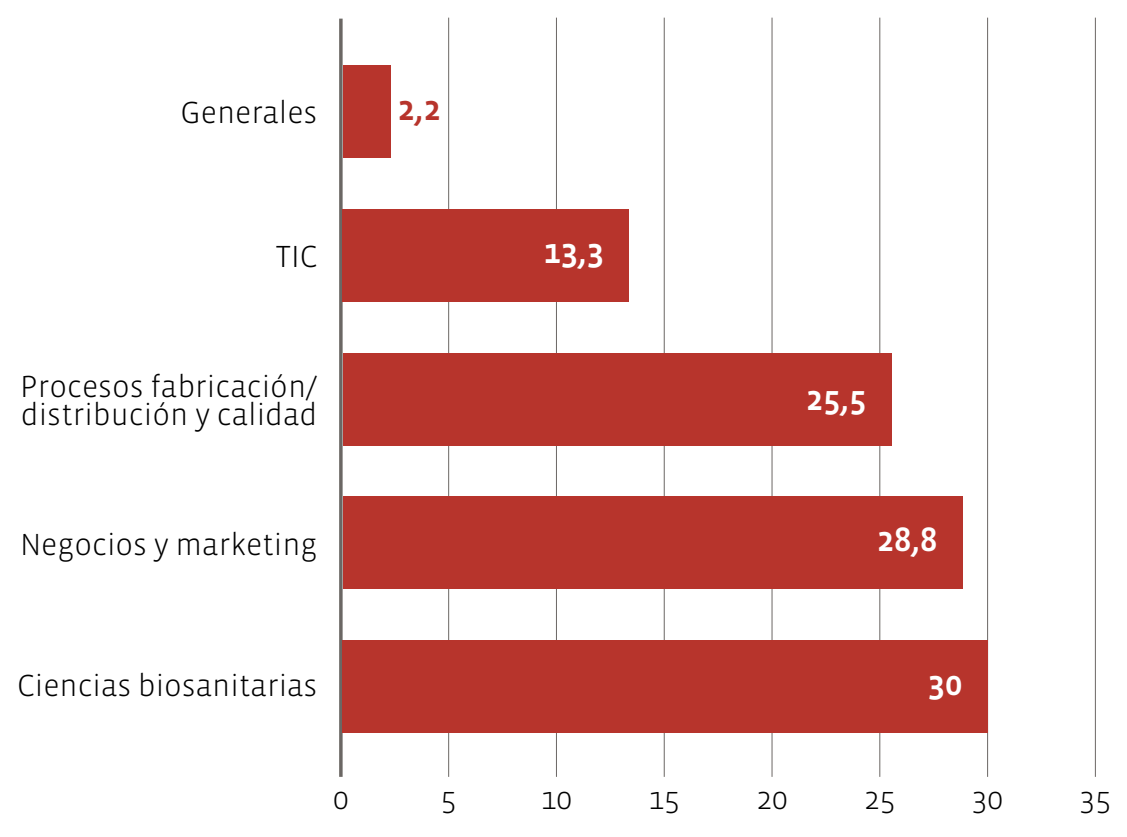

Tal como refleja el gráfico 1, el ámbito biosanitario ofrece el mayor porcentaje de anglicismos, seguido del terreno de los negocios y el marketing. A continuación, el área de los procesos de fabricación/distribución y los controles de calidad ocupa un tercer puesto, que va seguido del campo de las TIC. Dada la escasa diferencia porcentual que existe entre los ámbitos que ocupan los tres primeros lugares, se observa con claridad la multidisciplinariedad del profesional farmacéutico.

\subsection{Función de estos anglicismos en el campo de la farmacia}

Las razones que motivan a los profesionales del campo de las ciencias farmacéuticas a usar estas unidades léxicas anglicadas pueden ser variadas. Siguiendo la categorización de Rodríguez-González (1996) de las funciones de los anglicismos en español, podríamos señalar que podemos considerar que muchos de los vocablos empleados desempeñan una función referencial, cuyo objeto no es otro que el de cubrir un hueco o vacío semántico, al no existir un término equivalente en español para definir un determinado concepto. Para ilustrar, blister pack es un tipo de envasado, en plástico generalmente, donde se alojan medicamentos en forma de cápsulas o píldoras. En español no existe un término que exprese con exactitud este concepto. En otro campo como el del marketing, López Zurita (2018: 131) señala que sí existe una necesidad real lingüística para acudir al inglés en muchos casos. 
En segundo lugar, otros de los anglicismos desempeñan una función textual, que, como es sabido, puede implicar economía de la expresión. Hay ejemplos en los que el término en castellano es más largo que el inglés, como en el caso de retail para referirse a un tipo de ventas al detalle o al por menor. Lógicamente, el término retail es más corto y económico, desde el punto de vista lingüístico, para referirse a este tipo de ventas.

Por último, en otros casos, podemos considerar que desempeñan una función expresiva o estilística, pues, pese a que existe un término equivalente en español, se escoge la unidad léxica anglicada porque aporta otros valores, sentimientos o emociones que no contiene el término en español. Tal es el caso de pricing, que es usado pese a la existencia de un equivalente en español "tarificación”, "precio", o la expresión public affairs manager frente al español "jefe de asuntos públicos". Son términos que aportan cierto aire de internacionalismo e incluso esnobismo que no poseen sus equivalentes en español.

\section{Discusión}

Teniendo en cuenta las preguntas de investigación de partida de este estudio, se puede afirmar que, en efecto, sí que existe presencia del inglés en el campo de las ciencias de la farmacia. En 2006, Alcaraz Varó ya aportaba datos fehacientes sobre tal presencia. Más de una década más tarde, podemos continuar confirmando que continúa existiendo tal presencia y, que, con bastante probabilidad, vaya en alza.

Con respecto a la segunda pregunta, se puede confirmar que son los anglicismos no adaptados los más extendidos, con un $76,7 \%$ de la muestra recopilada. Los siguen los calcos del inglés al español con un $14,5 \%$ del total y, en menor medida, los híbridos, con un 6,6\%. Siguen dos únicos casos de anglicismos adaptados: emblistado y randomizado, con un 2,2\% del total de la muestra.

En lo que se refiere al grado de tecnicidad que presentan las unidades léxicas examinadas, se puede observar que los términos semitécnicos parecen ser los más extendidos, con un 47,7\% del total de la muestra, seguidos por el vocabulario puramente técnico, dentro del campo de las ciencias de la farmacia, con un 20\%, y, por último, por términos comunes (32,3\%), aunque también lógicamente empleados en el dominio de la farmacia.

La clasificación de los términos por campos semánticos ha demostrado que el campo de las ciencias biosanitarias aporta el mayor porcentaje, 30\%, seguido del terreno de los negocios y el marketing con un $28,8 \%$ de unidades léxicas, seguido de cerca por el terreno de los procesos de fabricación/distribución y gestión de calidad de los productos, con un 25,5\% del total, seguido por el campo de las TIC, con un 13,3\%, y, por último, por términos generales con un $2,2 \%$ del total. Como señalábamos anteriormente, el farmacéutico, además de profesional del medicamento, es gestor y empresario, de ahí la variedad de términos empleados. 
En lo que atañe a la función que desempeñan estos anglicismos en el campo de especialización de las ciencias farmacéuticas, se puede concluir que no hay una única función, sino, por el contrario, una combinación de varias. En el caso de algunos de los extranjerismos analizados, observamos una función textual; en otros, referencial, donde se trata de cubrir un hueco o vacío terminológico al no existir un equivalente en español que exprese con precisión un determinado concepto. También hallamos casos en que se trata de una función meramente expresiva o estilística, con la que el usuario del término tal vez pretende sonar más profesional o moderno.

\section{Conclusiones}

Este trabajo, a través de un análisis cualitativo, pretende arrojar luz acerca del impacto y de la influencia que el inglés ejerce no solo en la vida cotidiana de cualquier ciudadano, sino además en los campos o lenguajes más especializados y técnicos. Para concluir, con independencia de las razones que inducen al empleo de estos anglicismos, la realidad es que estas unidades léxicas anglicadas son empleadas en el campo de especialización de las ciencias farmacéuticas, lo que indica que, al igual que ocurre con otros muchos terrenos de especialización, la farmacia no es impermeable al empleo de anglicismos.

Se trata de un estudio que, con sus limitaciones, contribuye a arrojar luz sobre el tema en cuestión. Algunas propuestas de investigación futura incluirían una ampliación de la muestra, que podría abarcar un mayor número de revistas digitales y otro tipo de fuentes impresas. Asimismo, sería de gran interés complementar los resultados obtenidos de estas fuentes escritas con datos extraídos sobre el uso de estos anglicismos en el lenguaje oral. Esto se podría llevar a cabo mediante la realización de entrevistas a una muestra de farmacéuticos que confirmen si usan o no estos términos en su entorno profesional y/o en su vida cotidiana. Por último, también sería interesante comparar lo que ocurre con el uso de anglicismos entre profesionales de distintos países europeos como Alemania, Francia, Italia, Portugal, Polonia, así como en otros lugares del mundo tales como China, Japón, Brasil, Rusia, por mencionar solo algunos países.

\section{Bibliografía citada}

\subsection{Fuentes primarias}

www.correofarmaceutico.com

www.elfarmaceutico.es

www.infarmacias.es 


\subsection{Fuentes secundarias}

Acosta Artlles, Francisco Javier, y Alicia Bolaños Medina, 2017: "Anglicismos léxicos evitables en artículos científicos de salud mental”, Panace@ XVIII, 45, 3-11.

Alcaraz Ariza, María Ángeles, 1998: Anglicismos en el lenguaje de las ciencias de la salud. Tesis doctoral inédita, Universidad de Alicante.

Alcaraz Ariza, María Ángeles, 2002: “Los epónimos en medicina”, Ibérica 4, 55-73.

Alcaraz Varó, Enrique, 2000: El inglés profesional y académico, Madrid: Alianza.

Alcaraz Varó, Enrique, 2006: "Aproximación al lenguaje de la Farmacia”, Anales de la Real Academia Nacional de Farmacia 72, 343-360.

Alcaraz Varó, Enrique, 2008: "Language and health care: Food for thought", Panace@ IX, 28, 161-168.

Alcaraz Varó, Enrique, Alfonso Dominguez-Gil y Raquel Martinez Motos, 2007: Terminological Dictionary of Pharmaceutical Sciences (Inglés-Español, Spanis-English), Barcelona: Ariel.

ARIAS, Tomas D., 1999: Glosario de medicamentos: desarrollo, evaluación y uso, Washington D.C.: Organización Panamericana de la Salud.

De la Cruz, Isabel, y Cristina Tejedor, 2012: "Email o correo electrónico? Anglicisms in Spanish", RESLA 25 (1), 95-117.

Furiassı, Cristiano, Virginia Pulcini y Félix Rodriguez Gonzalez (eds.), 2012: The Anglicization of European Lexis, Amsterdam y Philadelphia: John Benjamins.

GutiérRez Rodilla, Bertha, 2005: El lenguaje de las ciencias, Madrid: Gredos.

López ZuRita, Paloma, 2018: "El uso de anglicismos en el contexto del vocabulario académico de Marketing”, Onomázein 39, 114-139.

Lorenzo Criado, Emilio, 1987: "Anglicismos en la prensa" en Actas de la Primera Reunión de Academias de la Lengua Española sobre el Lenguaje y los Medios de Comunicación, Madrid: Real Academia Española, 71-79.

LuJÁn-Garcia, Carmen, 2020: "The use of English initialisms and abbreviations in the field of pharmaceutical business communication in Spanish”, Terminology 26, 1, 60-81. 
Martínez-Motos, Raquel, 2011: "The Role of Interdisciplinarity in Lexicography and Lexicology" en Isabel Balteiro (ed.): New Approaches to Specialized English Lexicology and Lexicography, Newcastle upon Tyne: Cambridge Scholars Publishing, 3-15.

Navarro, Fernando, 2001: “El inglés, el idioma internacional de la medicina”, Panace@ 2, 3, 35-51.

Navarro, Fernando, 2002: "En pos de la verdadera causa de los anglicismos médicos", Ars Medica. Revista de Humanidades 1, 53-64.

Navarro, Fernando, y Javier Gonzalez de Dios, 2014. "Palabras y expresiones inglesas de traducción difícil o engañosa en investigación clínica, bioestadística y medicina basada en la evidencia”, Emergencias 26, 375-392.

Neumann, Natascha, 2013: La influencia de los anglicismos en el lenguaje sociosanitario español y las consecuencias para el papel del traductor especializado. Trabajo de fin de máster: Universidad de Alcalá.

Real Academia Española, 2014: Diccionario de la lengua española. DLE, 23. ${ }^{a}$ edición, Madrid [disponible en http://dle.rae.es/].

Rodriguez GonzÁlez, Félix, 1996: “Functions of Anglicisms in Contemporary Spanish”, Cahiers de Lexicologie 68, 1, 107-128.

Rodriguez González, Félix, 2017: Gran diccionario de anglicismos, Madrid: Arco Libros.

SegurA, Joaquín, 2001: "Los anglicismos en el lenguaje médico”, Panace@ 2, 3, 52-57.

\section{Anexo}

\section{TABLA 3}

Anglicismos no adaptados o crudos

ANGLICISMOS

NO ADAPTADOS

App

\section{USOS EN CONTEXTO \\ USOS EN CONTEXTO}

Quince soluciones. Un jurado. Tres finalistas y...

Un ganador: el equipo \#Lápices y su app para la

farmacia MyFarma (www.correofarmaceutico.

com, 15/03/2018)

\section{SIGNIFICADO O EQUIVA-}

LENTE EN ESPAÑOL

Aplicación informática 


\begin{tabular}{|c|c|c|}
\hline Area Manager & $\begin{array}{l}\text { A la vuelta, guiada por esta convicción decidió } \\
\text { matricularse en Diseño de Interiores en la Es- } \\
\text { cuela Superior de Diseño La Llotja de Barcelona, } \\
\text { y un tiempo después se apuntó en la prestigiosa } \\
\text { escuela de diseño Elisava, donde cursó un más- } \\
\text { ter en Retail Design mientras trabajaba como } \\
\text { ‘área manager’ para una cadena de parafarma- } \\
\text { cias (www.elfarmaceutico.es, 13/10/2014) }\end{array}$ & Gerente de área \\
\hline Benchmarking & $\begin{array}{l}\text { Según la experta de IMS, existen tres tipos de } \\
\text { benchmarking, el interno, el funcional y el } \\
\text { competitivo) (www.correofarmaceutico.com, } \\
\text { 04/07/2016) }\end{array}$ & $\begin{array}{l}\text { Medida estándar a la que una ac- } \\
\text { tividad, rendimiento, servicio o re- } \\
\text { sultado se puede comparar }\end{array}$ \\
\hline Big data & $\begin{array}{l}\text { Teva se sumerge en el ‘big data’ de la mano de } \\
\text { IBM (www.correofarmaceutico, 15/09/2015) }\end{array}$ & Gestión masiva de datos \\
\hline Blister pack & $\begin{array}{l}\text { Zaragozá también habló de investigar para de- } \\
\text { sarrollar nuevas formas de administración que } \\
\text { mejoren la toma de los medicamentos. "Tene- } \\
\text { mos blísters imposibles de abrir para una per- } \\
\text { sona mayor y antipsicóticos muy difíciles de } \\
\text { tomar por los enfermos", constata. (wWw.co- } \\
\text { rreofarmaceutico.com, 01/03/2018) }\end{array}$ & $\begin{array}{l}\text { Envase para manufacturados pe- } \\
\text { queños en soporte de cartón o car- } \\
\text { tulina (GDA) }\end{array}$ \\
\hline$B \log$ & $\begin{array}{l}\text { Un buen blog podría ser responsable de más del } \\
50 \% \text { de las ventas ‘on line’ (wWw.correofarma- } \\
\text { ceutico.com, 22/05/2017) }\end{array}$ & $\begin{array}{l}\text { Sitio web que incluye, a modo de } \\
\text { diario personal de su autor o au- } \\
\text { tores, contenidos de su interés, } \\
\text { actualizados con frecuencia y a } \\
\text { menudo comentados por su lecto- } \\
\text { res (DLE) }\end{array}$ \\
\hline Blue-box & $\begin{array}{l}\text { A efectos de impresión se mantendrá en el án- } \\
\text { gulo superior derecho de las caras principales } \\
\text { del embalaje exterior, o en la esquina superior } \\
\text { derecha de la blue box, según proceda. (www. } \\
\text { correofarmaceutico.com, 13/07/2017) }\end{array}$ & $\begin{array}{l}\text { Área recuadrada que contenga la } \\
\text { información nacional en los proce- } \\
\text { dimientos descentralizado y reco- } \\
\text { nocimiento mutuo }\end{array}$ \\
\hline $\begin{array}{l}\text { Business Develo- } \\
\text { pment Manager }\end{array}$ & $\begin{array}{l}\text { Montse Martí, hija del fundador y actual Busi- } \\
\text { ness Development de MartiDerm, dio un emo- } \\
\text { tivo discurso en el que no sólo recordó la figura } \\
\text { de su padre y su avanzada visión para ofrecer } \\
\text { soluciones dermatológicas eficaces desde } 1952 \\
\text { (www.elfarmaceutico.es, } 18 / 07 / 2017 \text { ) }\end{array}$ & Gerente de desarrollo de negocios \\
\hline Business Unit & $\begin{array}{l}\text { La jornada de hoy se ha abierto a las 10:00 h con } \\
\text { la celebración de una mesa redonda (Sala } 1 \text { ) en } \\
\text { la que Javier Altemir, business unit senior de Al- } \\
\text { mirall HealhtCare } \\
\text { (www.elfarmaceutico.es, 10/03/2016) }\end{array}$ & Unidad de negocio \\
\hline $\begin{array}{l}\text { Business Unit } \\
\text { Manager }\end{array}$ & $\begin{array}{l}\text { Rosa Vázquez Martín } \\
\text { Business Unit Manager. Gedeon Richter Ibérica } \\
\text { (www.elfarmaceutico.es, 27/11/2014) }\end{array}$ & Gerente de unidad comercial \\
\hline Checklist & $\begin{array}{l}\text { Ante una inspección, realizar una 'check list' y } \\
\text { disponer de formularios (wWw.correofarmaceu- } \\
\text { tico.com, 18/11/2013) }\end{array}$ & $\begin{array}{l}\text { Lista de datos que se anotan en } \\
\text { el control y seguimiento de algo } \\
\text { (GDA) }\end{array}$ \\
\hline
\end{tabular}




\begin{tabular}{ll}
\hline Checkpoint & Una alta carga mutacional puede ser predictiva \\
& de respuesta a pembrolizumab, un inhibidor \\
& del checkpoint PD-1, recientemente aprobado \\
& por la agencia reguladora estadounidense FDA \\
& en pacientes con tumores con inestabilidad \\
& de microsatélites con independencia del tipo \\
& histológico. (www.correofarmaceutico.com, \\
& 05/06/2018) \\
\hline Clinicaltrial & La Facultad de Farmacia de la Universidad CEU \\
management & San Pablo ha celebrado el acto de entrega de \\
& los certificados en Clinical Trials Management \\
& and Regulatory Compliance otorgados por The \\
& University of Chicago Graham School of Con- \\
& tinuing Liberal and Professional Studies a los \\
& alumnos de la quinta promoción del grado en \\
& Farmacia Bilingüe. (www.correofarmaceutico. \\
& com, 13/04/2016)
\end{tabular}

Clinical Research Hay una figura dentro del sector farmacéutico Assistant que ha experimentado un auge en los tiempos recientes es la del monitor de ensayos clínicos o clinical research assistant (CRA). (WWW.correofarmaceutico.com, 04/02/2013)

Cross Docking Con almacenes de cross docking en 15 puntos estratégicos desde los que STX Medical da cobertura a todo el territorio nacional. (www. imfarmacias.es, 15/05/2015)

Customer journey

Para lograrlo, se sigue la metodología del Customer Journey (viaje del cliente) en la botica, que va desde el escaparate, los planogramas, la teatralización, la indagación de necesidades, el consejo y el seguimiento (www.correofarmaceutico.com, 02/07/2018)

Data matrix Además, a efectos de codificación, el CN quedará incorporado a la información contenida en el código de producto incluido en el código de barras bidimensional (también denominado código datamatrix). (WWW.correofarmaceutico. com, 13/07/2017)

Data mining
Punto de control (GDA)

Gestión de ensayos clínicos

Monitor de ensayos clínicos

Sistema de distribución donde las unidades logísticas son recibidas en una plataforma de alistamiento y no son almacenadas sino preparadas para ser enviadas de la manera más inmediata

Es el trayecto que sigue un usuario desde el momento en que se plantea la posibilidad de adquirir un bien o servicio hasta que lo adquiere

Codificación de datos 2D

Extracción de información oculta y aplicable mediante análisis prospectivo automatizado de grandes bases de datos, consideramos más claras en español las traducciones del tipo de explotación de datos o prospección de datos (Navarro y González de Dios, 2014: 379) 
Endpoint

Export Manager
En cuanto a la fase clínica, la SEOM considera importante "que los ensayos clínicos incluyan endpoints secundarios (por ejemplo supervivencia libre de recaída, supervivencia libre de progresión, supervivencia global) que permitan estimar si la comparabilidad del biosimilar frente al anticuerpo de referencia en cuanto al objetivo primario se sostiene en endpoints de mayor importancia clínica para la supervivencia de los pacientes". (www.correofarmaceutico.com, 03/12/2015)

\begin{tabular}{ll}
\hline Export Manager & La incorporación de Francesc Rosell, que hasta \\
& la fecha era Export Manager de la compañía \\
& ITW Reagents (Panreac Applichem) en Barcelo- \\
& na, refuerza el destacado posicionamiento de \\
& Salvat en el área internacional. (wWW.correofar- \\
& maceutico.com, 22/01/2016)
\end{tabular}

Gold standard El tratamiento incluye cambios en el estilo de vida (deben evitarse el alcohol y otras drogas) ${ }^{31}$, y otros tratamientos específicos, como la terapia con presión positiva continua en la vía respiratoria (CPAP por sus siglas en inglés), que se considera el gold standard, los dispositivos orales y la cirugía, considerándose la traqueotomía el tratamiento quirúrgico primario en esta patología (www.elfarmaceutico.es, 04/04/2018)

Good Clinical

Practices

Al principio hay que viajar un poco por los hospitales, pero a medida que vas creciendo viajas menos y puedes especializarte en temas más regulatorios (regulatory affairs) o de calidad (dentro de las good clinical practices). (wWW. elfarmaceutico.es, 29/05/2017)

Federico Plaza, director de Government Affairs

Government Affairs Manager de Roche

(www.elfarmaceutico.es, 01/09/2016)
Se usa para designar, en los estudios clínicos, la variable predefinida que permite cuantificar el efecto de una intervención (Navarro y González de Dios, 2014: 380-381)

Gerente de exportaciones

Esta expresión inglesa, que no debe confundirse con standard gold (oro de ley), se ha puesto de moda en los dos últimos decenios para designar el mejor de los métodos diagnósticos disponibles o el mejor tratamiento disponible ( $\mathrm{Na}$ varro y González de Dios, 2014: 382)

'Prácticas clínicas correctas' o, como se hace en el entorno de la ONU (que incluye a organismos internacionales como la OMS, la FAO, el Unicef o la OEA), 'prácticas clínicas adecuadas'. (Navarro y González de Dios, 2014: 383)

Gerente de asuntos gubernamentales
Hashtag

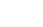

Todos los sujetos que han participado en el ensayo clínico podrían compartir sus vivencias una vez finalizado el programa, y éste podría seguirse de manera abierta mediante, por ejemplo, el uso de \#hashtags o grupos de trabajo ${ }^{3}$. (www.elfarmaceutico.es, 25/06/2018)

Hazard ratio
El estudio TERRAIN ha alcanzado su objetivo primario demostrando un aumento estadísticamente significativo en supervivencia libre de progresión (SLP) con enzalutamida frente a bicalutamida (Hazard Ratio $=0,44$; intervalo de confianza del 95\%, 0,34-0,57; p<0,0001). (www.elfarmaceutico.es, 19/01/2016)
Cadena de caracteres formada por una o varias palabras que constituyen una etiqueta, la cual va precedida de una almohadilla para que el usuario de las redes sociales la identifique de forma rápida (GDA)

Cociente de riesgo o de peligro (Navarro y González de Dios, 2014: 383) 


\begin{tabular}{ll}
\hline Items & La escala de sobrecarga del cuidador-test de \\
& Zarit mide el grado de sobrecarga subjetiva de \\
& los cuidadores, principalmente ancianos con \\
& algún grado de deterioro cognitivo. Consta de \\
& 22 ítems relacionados con las sensaciones del \\
& cuidador cuando cuida a otra persona, cada \\
& uno de los cuales se puntúa en un gradiente de \\
& frecuencia que va desde 1 (nunca) a 5 (casi siem- \\
& pre). Es autoadministrada \\
& (www.elfarmaceutico.es, 26/01/2015)
\end{tabular}

Joint venture En este sentido, Navarro afirmó que Pensa "no es una compañía multinacional, sino internacional”, ya que está abierta a cualquier tipo de relación con otras compañías (adquisición, joint-venture, alianzas) manteniendo las señas de identidad de las mismas, como ocurre con toLife y Breckenridge, que conservan su nombre original. (www.correofarmaceutico.com, 21/06/2014)

\begin{tabular}{ll} 
Keyaccount & Key Account Manager, investigación preclíni- \\
manager & ca: gestor de cuentas para gestionar y detectar \\
oportunidades comerciales de la investigación & preclínica de una compañía. \\
& (www.elfarmaceutico.es, 11/04/2013) \\
\hline Kit & «unas 400 familias de las comunidades más \\
& afectadas por los huracanes Irma y María en \\
& República Dominicana recibieron 400 kits hi- \\
& giénicos y de alimentos, 7.500 botellas de agua \\
& potable y 200 kits de protección y cobijo, entre \\
& otros enseres, gracias al apoyo de las unidades \\
& móviles de salud de MoscHTA, nuestra socia \\
& local en el país», afirma Peris, que agradece el \\
& esfuerzo y compromiso de todas las entidades \\
& miembros del Fondo de Emergencias. \\
& (www.elfarmaceutico.es, 09/01/2018)
\end{tabular}

Lock-up STA Trust ha asumido un compromiso de no disposición o "lock-up" de 2 años con respecto al 50 euros de las acciones y de cuatro años respecto al restante 50 euros de las acciones. (www.correfarmaceutico.com, 17/05/2017).

Low dose

Isdin lanza Itragerm 50mg, el primer itraconazol low dose con biodisponibilidad mejorada (www.infarmacias.es, 12/11/2014)

Market Access Department/ manager
Unidades

Sociedad formada por dos o más empresas que se unen para la realización de un proyecto o negocio determinado (GDA)

\section{Gestor de grandes cuentas}

Equipo

Periodo durante el cual no es posible realizar una determinada operación (eleconomista.es)

Dosis baja

Departamento o persona que optimiza el proceso que se ha de seguir en la industria farmacéutica para hacer llegar al mercado (y por tanto a los/las pacientes) uno de sus fármacos 


\begin{tabular}{|c|c|c|}
\hline Non-sense & $\begin{array}{l}\text { El medicamento está destinado a pacientes } \\
\text { deambulantes mayores de } 5 \text { años que padezcan } \\
\text { la mutación “non sense”( } \text { sin sentido). (Www.co- } \\
\text { rreofarmaceutico.com, } 16 / 11 / 2016 \text { ) }\end{array}$ & Sin sentido \\
\hline Odds ratio & $\begin{array}{l}\text { Un segundo aspecto que merece la pena desta- } \\
\text { car y de mucha relevancia se refiere a la inter- } \\
\text { pretación que hace de la odds ratio. (www.elfar- } \\
\text { maceutico.es, } 13 / 03 / 2015 \text { ) }\end{array}$ & $\begin{array}{l}\text { Razón (o cociente) de posibilida- } \\
\text { des (Navarro y González de Dios, } \\
\text { 2014: 386) }\end{array}$ \\
\hline One-to-one & $\begin{array}{l}\text { Se trata de un servicio gratuito que el COFM } \\
\text { ofrece a los colegiados y a los nuevos farmacéu- } \\
\text { ticos, consistente en una consultoría personali- } \\
\text { zada sobre las opciones de empleo en diversos } \\
\text { sectores y funciones del sector de la salud, in- } \\
\text { cluyendo asesoría one-to-one sobre la realiza- } \\
\text { ción de currículums, entrevistas, etc. } \\
\text { (www.elfarmaceutico.es, 03/07/2017) }\end{array}$ & Personalizado \\
\hline Open source & $\begin{array}{l}\text { Las tecnologías open source se convertirían en } \\
\text { el motor de la nueva generación de proceso de } \\
\text { datos y permitirían análisis más amplios de for- } \\
\text { ma más rápida. (www.correofarmaceutico.com, } \\
\text { 12/07/2016) }\end{array}$ & $\begin{array}{l}\text { Herramientas informáticas de ac- } \\
\text { ceso abierto y libres de pago }\end{array}$ \\
\hline $\begin{array}{l}\text { Ophthalmolo- } \\
\text { gical tested }\end{array}$ & $\begin{array}{l}\text { 10. No pica en los ojos (ophtalmological tested). } \\
\text { (wwW.elfarmaceutico.es, 16/03/2015) }\end{array}$ & Testado oftalmológicamente \\
\hline Partner & $\begin{array}{l}\text { Alloga será ‘partner’ en exclusiva para distribuir } \\
\text { la vacuna para la fiebre tifoidea } \\
\text { (www.correfarmaceutico.com, 27/04/2015) }\end{array}$ & $\begin{array}{l}\text { Persona que toma parte en una ac- } \\
\text { tividad comercial (GDA) }\end{array}$ \\
\hline Peer Monitoring & $\begin{array}{l}\text { Cadena de mando y «peer monitoring» } \\
\text { (www.elfarmaceutico.es, 12/12/2012) }\end{array}$ & Control o seguimiento \\
\hline Pellets & $\begin{array}{l}\text { Los productos estrella de la fábrica maña, que } \\
\text { es referencia dentro del grupo en tecnolo- } \\
\text { gías de pellets (cápsulas con gránulos), son el } \\
\text { omeprazol, el lansoprazol, la atorvastatina y } \\
\text { el pantoprazol. (www.correfarmaceutico.com, } \\
\text { 11/10/2014) }\end{array}$ & $\begin{array}{l}\text { Forma farmacéutica solida prepa- } \\
\text { rada para su implantación debajo } \\
\text { de la piel con el fin de obtener una } \\
\text { absorción lenta y sostenida }\end{array}$ \\
\hline Pipeline & $\begin{array}{l}\text { En su pipeline destacan LAS41008 (DMF) y tildra- } \\
\text { kizumab, ambos para psoriasis; P3058, para oni- } \\
\text { comicosis; P3073, para psoriasis de las uñas, y } \\
\text { P3074, que es una nueva formulación tópica de } \\
\text { finasteride, para alopecia androgenética. (WWW. } \\
\text { correfarmaceutico.com, 27/02/2017) }\end{array}$ & $\begin{array}{l}\text { Línea o tubería que conduce el pe- } \\
\text { tróleo a larga distancia, desde los } \\
\text { campos de extracción hasta las re- } \\
\text { finerías o los puertos de embarque } \\
\text { (GDA) }\end{array}$ \\
\hline Pivotal & $\begin{array}{l}\text { El estudio pivotal ADVANCE demostró un perfil } \\
\text { de seguridad similar al resto de los interfero- } \\
\text { nes en el tratamiento de la esclerosis múltiple } \\
\text { remitente recurrente. (www.elfarmaceutico.es, } \\
02710 / 2015 \text { ) }\end{array}$ & $\begin{array}{l}\text { Central, fundamental o esencial; p. } \\
\text { ej.: pivotal study o pivotal trial (Na- } \\
\text { varro y González de Dios, 2014: } 387 \text { ) }\end{array}$ \\
\hline
\end{tabular}




\begin{tabular}{|c|c|c|}
\hline Premium price & $\begin{array}{l}\text { Como consecuencia, la Administración valoró } \\
\text { esta apuesta y le concedió un pequeño pre- } \\
\text { mium price, puesto que demostró que no era } \\
\text { un genérico al uso". (www.correfarmaceutico. } \\
\text { com, 01/03/2018) }\end{array}$ & Reconocimiento o premio \\
\hline Pricing & $\begin{array}{l}\text { En BMS también trabajó como mánager de Price } \\
\text { \& Market Access, directora asociada de Pricing \& } \\
\text { Reimbursement para Europa y mánager de Mar- } \\
\text { ket Access en Cardiovascular y Metabolismo. } \\
\text { (www.elfarmaceutico.es, 11/09/2015) }\end{array}$ & $\begin{array}{l}\text { Tarificación o establecimiento de } \\
\text { precios }\end{array}$ \\
\hline Prime Time & $\begin{array}{l}\text { Médicos insisten en adelantar la hora del ‘prime } \\
\text { time’ televisivo en España (wWw.correofarma- } \\
\text { ceutico.com, 14/01/2017) }\end{array}$ & Hora de máxima audiencia \\
\hline Product manager & $\begin{array}{l}\text { Asílo cree Susana Barragán, directora de Adecco } \\
\text { Farma-Química, según la cual ambos demandan } \\
\text { perfiles muy parecidos: "Delegados de ventas } \\
\text { y marketing, product manager, market access, } \\
\text { MSL (Medical Scientific Liaison), operaciones y } \\
\text { logística, técnicos y analistas especializados...". } \\
\text { (www.correfarmaceutico.com, 29/02/2016) }\end{array}$ & $\begin{array}{l}\text { En grandes empresas, responsable } \\
\text { de ventas de uno o más productos } \\
\text { (GDA) }\end{array}$ \\
\hline $\begin{array}{l}\text { Public Affairs } \\
\text { Manager }\end{array}$ & $\begin{array}{l}\text { Área Corporate (Reputación y Posicionamiento } \\
\text { Corporativo, RSE y Ciudadanía Corporativa, Cri- } \\
\text { sis \& Issues, Public Affairs, Comunicación Finan- } \\
\text { ciera, Investors Relations y Buen Gobierno, Re- } \\
\text { laciones Institucionales, Corporativas \& Market } \\
\text { Access) } \\
\text { (www.elfarmaceutico.es, 24/02/2015) }\end{array}$ & Gerente de asuntos públicos \\
\hline $\begin{array}{l}\text { Quality and com- } \\
\text { pliance manager }\end{array}$ & $\begin{array}{l}\text { Precisamente, con respecto al contrato, Ana } \\
\text { González, quality \& compliance manager de } \\
\text { Janssen, ha resaltado la importancia de que } \\
\text { en un futuro se contemple un contrato único, } \\
\text { ya que "el principal cuello de botella en los en- } \\
\text { sayos clínicos siempre han sido los contratos } \\
\text { con los centros. (www.correfarmaceutico.com, } \\
\text { 30/11/2016). }\end{array}$ & $\begin{array}{l}\text { Gerente de calidad y cumplimien- } \\
\text { to }\end{array}$ \\
\hline Ranking & $\begin{array}{l}\text { La página líder de este ranking no es de venta } \\
\text { de medicamentos o productos sanitarios sino } \\
\text { que es la estadounidense Drugs.com } \\
\text { (www.correofarmaceutico.com, 07/05/2018) }\end{array}$ & $\begin{array}{l}\text { Clasificación de mayor a menos, } \\
\text { útil para establecer criterios de va- } \\
\text { loración (DLE) }\end{array}$ \\
\hline Ready-made & $\begin{array}{l}\text { El establecimiento del catering en las compa- } \\
\text { ñías aéreas y el ready-made y pret-â-porter de la } \\
\text { moda sirvieron como inspiración a este cambio } \\
\text { de pensamiento. (www.correfarmaceutico.com, } \\
28 / 06 / 2010 \text { ) }\end{array}$ & $\begin{array}{l}\text { Objeto fabricado en serie o parte } \\
\text { de una máquina en desuso que se } \\
\text { expone como obra de arte (GDA) }\end{array}$ \\
\hline
\end{tabular}




\begin{tabular}{ll}
\hline Real-world & Flatiron Health, empresa privada de tecnología \\
evidence & y servicios de salud con sede en Nueva York (Es- \\
& tados Unidos), es uno de los líderes del merca- \\
& do en software de registro de salud electrónico \\
& específico de Oncología, así como experto en \\
& conservación y desarrollo de ensayos en vida \\
& real (real-world evidence) para la investigación \\
& del cáncer. (www.correofarmaceutico.com, \\
& $16 / 02 / 2018)$
\end{tabular}

Regulatory Barragán cree que los laboratorios en España sufren una falta de candidatos cualificados, pero no de manera muy significativa. "La balanza estaría algo descompensada por motivos vinculados a la fuga de talento a Europa o Estados Unidos, por el déficit de perfiles de desarrollo de protocolos en la industria y en las áreas de Regulatory y calidad...", explica. (www.correfarmaceutico.com, 29/02/2016).

\begin{tabular}{|c|c|}
\hline Reimbursement & $\begin{array}{l}\text { En BMS también trabajó como mánager de Price } \\
\text { \& Market Access, directora asociada de Pricing \& } \\
\text { Reimbursement para Europa y mánager de Mar- } \\
\text { ket Access en Cardiovascular y Metabolismo. } \\
\text { (www.elfarmaceutico.es, 11/09/2015) }\end{array}$ \\
\hline Retail & $\begin{array}{l}\text { Farmacéutica especializada en diseño de in- } \\
\text { teriores y retail (wWw.elfarmaceutico.es, } \\
\text { 14/10/2014) }\end{array}$ \\
\hline Screening & $\begin{array}{l}\text { En el ámbito de Farmacia Comunitaria, el pri- } \\
\text { mer premio ha sido para el trabajo «Técnica de } \\
\text { screening en orina para la detección de cistitis } \\
\text { no complicada», realizado por Sara Benadel y } \\
\text { Paula Franch, bajo la dirección de la profesora } \\
\text { María Rubio, de la Unidad de Coordinación Do- } \\
\text { cente Barcelona Norte (www.elfarmaceutico.es, } \\
25 / 04 / 2018 \text { ) }\end{array}$ \\
\hline Senior consultant & $\begin{array}{l}\text { Una situación que corrobora Álex Álvarez, se- } \\
\text { nior consultant de Randstad Professionals, se- } \\
\text { gún el cual "se busca un perfil de delegado de } \\
\text { visita consultiva orientada a resultados con co- } \\
\text { nocimientos científicos y de trade marketing". } \\
\text { (www.correfarmaceutico.com, 29/02/2016) }\end{array}$ \\
\hline $\begin{array}{l}\text { Senior Product } \\
\text { Manager }\end{array}$ & $\begin{array}{l}\text { Como explicó Miriam Mañas, product manager } \\
\text { de la compañía, en una presentación de sus } \\
\text { productos realizada en Madrid, en SVR se tra- } \\
\text { baja tanto en una línea más dermatológica de } \\
\text { productos para patologías, como puede ser una } \\
\text { piel atópica o una piel con tendencia acneica, } \\
\text { como en líneas más cosméticas. } \\
\text { (www.elfarmaceutico.es, } 17 / 11 / 2016 \text { ) }\end{array}$ \\
\hline
\end{tabular}

Ensayos en vida real

Que atiende a algún tipo de regulación o normativa

Gerente de reembolsos

Punto de ventas

Selección o cribado en un proceso clínico (Navarro y González de Dios, 2014: 389)

Asesor con experiencia

Gerente de productos 


\begin{tabular}{|c|c|c|}
\hline Software & $\begin{array}{l}\text { Según informó Alberto Zamora, del Hospital de } \\
\text { Blanes, en Gerona, en la jornada de adherencia } \\
\text { celebrada la semana pasada en Madrid, "este } \\
\text { software está basado en la Guía Europea de } \\
\text { Dislipemias del } 2011 \text { (www.correofarmaceutico. } \\
\text { com, } 21 / 11 / 2016 \text { ) }\end{array}$ & $\begin{array}{l}\text { Conjunto de programas, instruc- } \\
\text { ciones y reglas informáticas para } \\
\text { ejecutar ciertas tareas en una } \\
\text { computadora(DLE) }\end{array}$ \\
\hline Source data & $\begin{array}{l}\text { Comprobar que los datos registrados de los } \\
\text { pacientes participantes son los correctos (mo- } \\
\text { nitorización y Source Data Verification). (wWw. } \\
\text { elfarmaceutico.es, } 11 / 04 / 2013 \text { ) }\end{array}$ & $\begin{array}{l}\text { Datos originales (Navarro y Gonzá- } \\
\text { lez de Dios, 2014: } 390 \text { ) }\end{array}$ \\
\hline Stakeholder & $\begin{array}{l}\text { Hace casi un año se aprobaba en España el Real } \\
\text { Decreto 1090/2015 de ensayos clínicos. Con esta } \\
\text { norma, se adelantó a otros Estados miembro en } \\
\text { la aplicación de la normativa europea. "Desde } \\
\text { que se implementó, tiene un saldo positivo para } \\
\text { todos los stakeholders involucrados" (wWW.co- } \\
\text { rrefarmaceutico.com, 30/11/2016) }\end{array}$ & Parte interesada \\
\hline Stand & $\begin{array}{l}\text { Las marcas integrantes del grupo, A-Derma, } \\
\text { Ducray, Dexeryl, Eau Thermale Avène, Klorane, } \\
\text { Elancyl, Galénic y René Furterer, estarán repre- } \\
\text { sentadas en un stand como espacio de interés } \\
\text { para el visitante. } \\
\text { (www.elfarmaceutico.es, 12/03/2018) }\end{array}$ & Puesto en una feria \\
\hline Start-up unit & $\begin{array}{l}\text { Ha señalado Marta Maislan, start up unit ma- } \\
\text { nager de Roche y moderadora de un simposio } \\
\text { sobre investigación clínica celebrado en el mar- } \\
\text { co del XII Congreso Nacional de la Asociación de } \\
\text { Medicina de la Industria Farmacéutica (Amife) } \\
\text { (www.correfarmaceutico.com, 30/11/2016) }\end{array}$ & $\begin{array}{l}\text { Empresa emergente, generalmen- } \\
\text { te asociada a la tecnología, que de- } \\
\text { sarrolla productos de gran innova- } \\
\text { ción, ofrece una alta rentabilidad } \\
\text { y exige una inversión limitada de } \\
\text { capital y trabajo (GDA) }\end{array}$ \\
\hline Stock & $\begin{array}{l}\text { No respetar el 'stock' mínimo abre la puerta a } \\
\text { sanciones en } 5 \text { autonomías (wWw.correofarma- } \\
\text { ceutico.com, 27/11//2017) }\end{array}$ & $\begin{array}{l}\text { Cantidad de cosas, especialmente } \\
\text { mercancías, que se tienen en de- } \\
\text { pósito para darles salida o venta } \\
\text { (GDA) }\end{array}$ \\
\hline Supply chain & $\begin{array}{l}\text { Elisenda Bordas Colom Directora de Farmacias } \\
\text { y Supply Chain. Sanofi España (www.elfarma- } \\
\text { ceutico.es, 27/11/2014) }\end{array}$ & $\begin{array}{l}\text { Responsable de hacer que fluya la } \\
\text { cadena de suministro o abasteci- } \\
\text { miento en una empresa }\end{array}$ \\
\hline Test & $\begin{array}{l}\text { Entre los hechos más relevantes de } 2017 \text { cabe } \\
\text { destacar la gestión llevada a cabo en Europa en } \\
\text { relación con la directiva del test de proporcio- } \\
\text { nalidad o la participación en el Sistema Espa- } \\
\text { ñol de Verificación de Medicamentos (SEVEM). } \\
\text { (www.elfarmaceutico.es, } 26 / 06 / 2018 \text { ) }\end{array}$ & $\begin{array}{l}\text { Prueba destinada a evaluar cono- } \\
\text { cimientos o aptitudes (DLE) }\end{array}$ \\
\hline Trade marketing & $\begin{array}{l}\text { Una situación que corrobora Álex Álvarez, se- } \\
\text { nior consultant de Randstad Professionals, se- } \\
\text { gún el cual "se busca un perfil de delegado de } \\
\text { visita consultiva orientada a resultados con co- } \\
\text { nocimientos científicos y de trade marketing". } \\
\text { (www.correfarmaceutico.com, 29/02/2016) }\end{array}$ & Marketing de comercio \\
\hline
\end{tabular}


Unit-dose
El término original unit-dose es el que acuña en inglés este envasado. Se debe traducir al español como dosis unitarias, como igualmente se traduce al portugués, francés e italiano. Traducirlo por unidosis ha sido por precipitación, ignorancia de su construcción morfosemántica y hasta por pereza mental. (www.correfarmaceutico.com, 28/06/2010)

Water proof O waterproof que indican que el preparado resiste 80 minutos de inmersión en el agua. (www. correofarmaceutico.com, 16/06/2015)
Hay propiedades como water resistant que implica que no pierde su capacidad protectora después de 40 minutos de inmersión en el agua. (www.correofarmaceutico.com, 16/06/2015)
Método de empaquetado y administración de medicación a través de una dosis indiviual

Producto cosmético que resiste el agua (GDA)

Que resiste el agua

\section{TABLA 4}

Anglicismos adaptados

\section{ADAPTADOS USOS EN CONTEXTO SIGNIFICADO EN ESPAÑOL}

Emblistado La industria está haciendo grandes esfuerzos por mejorar sus procesos. Un ejemplo es la introducción de la última tecnología en el emblistado de comprimidos de Cinfa. (CF) (www.correfarmaceutico.com, 02/03/2017)

Randomizado Este ensayo fase III pivotal y randomizado analiza la eficacia de Zepsyre ${ }^{\circledR}$ en combinación con doxorubicina en comparación con el tratamiento estándar en esta indicación. (www.correofarTipo de envase, generalmente de plástico transparente, con una cavidad en forma de ampolla donde se aloja el producto

Aleatorio, al azar, casual o fortuito, según el contexto (Navarro y González de Dios, 2014: 388) maceutico.com, 26/06/2018)

\section{TABLA 5}

Anglicismos híbridos

\section{HÍBRIDOS USOS EN CONTEXTO \\ SIGNIFICADO EN ESPAÑOL}

Farmacia on line

Un tribunal de Montana (Estados Unidos) ha impuesto una multa de 34 millones de dólares a Canada Drugs, farmacia on line, por vender medicamentos falsificados en territorio estadounidense. (www.correofarmaceutico.com, 05/07/2018)
Farmacia que vende medicamentos a distancia a través de internet. 
Gestión de

back-up

Medicamen-

to Me-Too glamento de desarrollo de la Ley Orgánica de Protección de Datos exige, en sus artículos 94 y 102, la realización de copias de respaldo y de recuperación de los ficheros automatizados que contengan datos de carácter personal. Una copia de respaldo o backup es la copia de un fichero automatizado en un soporte que posibilite su recuperación. (www.correofarmaceutico. com, 15/02/2016)

Para los me-too, el beneficio que se obtendrá va a estar relacionado con su capacidad competitiva más que con una necesidad del mercado", destaca Concha Álvarez, de Roche (www.correfarmaceutico.com, 05/02/2007)
El RD 1.270/2007, por el que se aprueba el Re-

Copia o reproducción que contiene una duplicación de un conjunto de datos y que se guardan como seguridad en caso de pérdida o destrucción (GDA)

Productos de imitación que tienen resultados clínicos más o menos idénticos a los de medicamentos existentes. Son compuestos muy similares estructuralmente a medicamentos ya conocidos que tienen un mecanismo de acción idéntico, con tan solo unas diferencias farmacológicas

La utilización de medicamentos fuera de ficha técnica ción 'off-label'

(wWw.correfarmaceutico.com, 11/09/2017)

Prescripción El bajo soporte científico pesa sobre la prescripofflabel

Test de legibilidad Autorización: la industria, preocupada por los test de legibilidad y el Braille (www.correfarmaceutico.com, 09/06/2008)

Es un test que permite demostrar que el entrevistado puede localizar la información en el prospecto fácilmente, que la entiende y que puede explicarla con sus propias palabras

Consultor senior La clase magistral del consultor Sénior en Nexe Vicenç Dopacio fue seguida por farmacéuticos Consultor con experiencia que, preocupados por los cambios del mercado y alertados por los nuevos modelos de negocio en el sector (www.elfarmaceutico.es, 03/03/2012)

\section{TABLA 6}

Calcos

\section{CALCOS} USOS EN CONTEXTO

Respecto al comercio paralelo, promovido por algunos países como Alemania, cuyo gobierno incentiva a las oficinas de farmacia la dispensación de medicamentos importados de otros países, Calduch subrayó que, aunque en principio son lícitas desde el punto de vista legal, las cooperativas deben tener como prioridad que el destino del producto a distribuir sean las oficinas de farmacia, con el fin de atender la demanda sanitaria de la población del territorio nacional. (www.elfarmaceutico.es, 27/03/2015)

\section{SIGNIFICADO EN ESPAÑOL}

Introducción de medicamentos en un mercado distinto al que habían sido comercializados previamente, lo que provoca casos de desabastecimiento de determinados productos en los mercados de origen y competencia 
co-pago

$\langle$ Co-payment $\rangle$

Coste-eficiencia
〈cost-efficiency

〈cost-efficiency〉

definida $<$ defined

daily dose $>$ (DDD)

AESEG celebró en el Senado el encuentro «20 años de genéricos en España» (www.elfarmaceutico.es, 02/02/2018)

Especificaciones

〈specifications〉

En lugar de generar un debate auténtico y nuclear, se desenfocó hacia quién debería pagar por ellos. Si los ciudadanos directamente a través del co-pago, o si debería ser a través del Sistema Nacional de Salud...

(www.elfarmaceutico.es, 28/09/2015)

Educación, información, diagnóstico precoz, infección oculta, comorbilidades, perfiles del paciente, profilaxis preexposición (PrEP) y terapia posexposición, es decir, todas las medidas coste-eficaces, deben priorizarse en el abordaje de VIH en España, según los expertos reunidos en la conferencia Vivir con el VIH, celebrada en Madrid de la mano de The Economist Events, con la colaboración de Gilead Sciences. (www. correfarmaceutico.com, 18/07/2017)

El nuevo sistema para calcular los precios de referencia, basado en el coste/tratamiento/día (CTD) y en la dosis diaria definida (DDD), busca una bajada forzada de los precios que produce graves errores.

(www.elfarmaceutico.es, 06/03/2014)

Conlleva una gran formación del equipo que va a llevar a cabo este servicio ya que es un ámbito con muchas referencias y marcas y cada uno tiene sus especificaciones (www.elfarmaceutico.es, 27/06/2016)

Genérico 〈generic>
Sistema de pago compartido de un medicamento donde un porcentaje es aportado por la Seguridad Social y otro porcentaje por el paciente

Procedimiento a través del cual se busca el camino más económico y expedito para alcanzar un objetivo; se trata de obtener el máximo rendimiento de un volumen determinado de recursos

Dosis de mantenimiento promedio en la principal indicación de un fármaco en pacientes adultos

Documentos en los cuales se definen las normas, exigencias y procedimientos a ser empleados y aplicados en todos los medicamentos, trabajos de construcción de obras, elaboración de estudios, fabricación de equipos

Según la OMS, un medicamento genérico es aquel que se vende bajo la denominación del principio activo que incorpora, suficientemente bioequivalente a la marca original, es decir, igual en composición y forma farmacéutica y con muy parecida biodisponibilidad que la misma.

Poner en funcionamiento o aplicar métodos, medidas, etc., para llevar algo a cabo (DLE)
Implementar

〈implement
Valdés explicó que las entidades de distribución ya han dado los primeros pasos para implantar el sistema, al iniciar la descarga de la documentación para desarrollar e implementar las interfaces de programación necesarias para conectarse con SEVeM y añadió que la disponibilidad del sistema con funcionalidad completa está prevista para diciembre de 2018. (www.elfarmaceutico.es, 15/03/2018) 
Medicamento huérfano <orphan medicine>
La inversión para desarrollar un medicamento huérfano suele ser superior a los 1.500 millones de euros.

(www.elfarmaceutico.es, 11/02/2015)

No desarrollado ampliamente por la industria farmacéutica por razones financieras, ya que van destinados a un reducido grupo de pacientes, y que, sin embargo, responden a necesidades de salud pública.

Monitorización

Abbott ha recibido la Marca CE (Conformidad <monitoring> Europea) para el Sistema Flash de monitorización de glucosa FreeStyle Libre indicado para niños y adolescentes con diabetes de 4 a 17 años. (www.elfarmaceutico.es, 16/02/2016)

Prescripción fuera de indicación <offlabel prescription>

La Comisión Regional de Farmacia y Terapéutica (CRFT), desde su constitución en 2012, ha garantizado el uso de medicamentos fuera de su indicación a más de 600 pacientes que precisaban utilizar fármacos en condiciones diferentes a las establecidas en la ficha técnica (www. imfarmacias.es, 18/07/2016)

Reportar <report $>$

Susana Vilas asume el liderazgo de esta área y se responsabiliza de desarrollar la estrategia integral de comunicación del Grupo, así como de gestionar su imagen corporativa. Vilas forma parte del Comité de Dirección del Grupo Indukern y reportará a Raúl Díaz-Varela, vicepresidente ejecutivo del Grupo. (www.elfarmaceutico.es, 08/02/2013)

Servicio nacional de salud <National Health Service>
De esta manera, los $A B S{ }^{\circledR}$ Pants se incorporan al catálogo de productos reembolsados por el Servicio Nacional de Salud con 9 referencias adaptándose a las distintas necesidades de los pacientes incontinentes.

(www.elfarmaceutico.es, 16/02/2017)
Control o seguimiento

Medicamento que se administra fuera de la indicación establecida

Transmitir, comunicar, dar noticia. U. m. en Am (DLE)
Conjunto coordinado de los servicios de salud de la Administración del Estado y los servicios de salud de las comunidades autónomas que integra todas las funciones y prestaciones sanitarias que, de acuerdo con la ley, son responsabilidad de los poderes públicos

\section{Tipificación (DLE)}

Proceso mediante el cual se realiza una actividad de manera standard o previamente establecida
Estandarización 〈standardization〉
También se utiliza en forma de extracto fluido (1:1) (0,5 mL, tres veces al día), o en forma de extractos secos estandarizados: cápsulas o comprimidos (de 120 a 240 mg/día, en 2-3 tomas); los mejores extractos estandarizados hacen referencia a una estandarización a partir de los flavonoides y lactonas terpénicas con un 24 y un $6 \%$, respectivamente. (www.elfarmaceutico.es, 18/06/2014) 